\title{
HLA-A*2402-Restricted VEGFR1 Peptide Vaccine
}

National Cancer Institute

\section{Source}

National Cancer Institute. HLA-A*2402-Restricted VEGFR1 Peptide Vaccine. NCI

Thesaurus. Code C77869.

A cancer vaccine containing the HLA-A*2402-restricted vascular endothelial growth factor receptor 1 (VEGFR1) peptide epitope with potential immunostimulatory and antitumor activities. Upon administration, HLA-A*2402-restricted VEGFR1 peptide vaccine may stimulate a cytotoxic $T$ lymphocyte $(C T L)$ response against tumor cells expressing VEGFR 1 peptide, resulting in tumor cell lysis and decreased tumor growth. HLA-A 2402 is an MHC class I molecule that presents antigenic peptides to CD8+ T cells; epitope design restricted to epitopes that bind most efficiently to HLA-A*2402 may improve antigenic peptide immunogenicity. 